J. Clin. Chem. Clin. Biochem

Vol. 28, 1990, pp. 453-458

(C) 1990 Walter de Gruyter \& Co. Berlin $\cdot$ New York

\title{
Comparison of Two Immunoinhibitory Methods with Agarose Gel-Electrophoresis for Measuring the MB Isoenzyme of Creatine Kinase in Serum from Cases of Suspected Myocardial Infarction
}

\author{
By A. Hadberg ${ }^{1}, C$. Hassager ${ }^{1}$, P. Hildebrandt ${ }^{2}$ and C. Christiansen ${ }^{1}$ \\ ${ }^{1}$ Department of Clinical Chemistry \\ 2 Department of Cardiology \\ Glostrup Hospital, University of Copenhagen, Denmark
}

(Received October 26, 1989/April 9, 1990)

Summary: Two immunoinhibitory methods for measuring creatine kinase-MB (a dry chemical and a wet chemical method) were compared with the commonly used agarose gel electrophoretic method, using 563 serum samples from 235 patients with suspected acute myocardial infarction. Comparison of the electrophoretic and the dry chemistry methods showed the linear relationship: electrophoretic method $=-6.5 \mathrm{U} / 1+1.22 \times$ dry chemistry method, $r=0.943$. For the wet chemistry method the relationship was: electrophoretic method $=-7.2 \mathrm{U} / 1+1.19 \times$ wet chemistry method, $\mathrm{r}=0.854$. Parallel determinations of total creatine kinase were also done and the methods were virtually identical in performance.

Compared with the electrophoretic method (which showed a $15 \%$ prevalence of acute myocardial infarction), these classifications showed sensitivities of 0.92 and 0.67 and specificities of 0.94 and 0.99 (dry and wet chemistry, respectively; using methods recommended by the manufacturers). After optimization of discriminators the sensitivity was increased to $0.94 / 0.92$ and the specificity to $0.99 / 0.99$.

\section{Introduction}

Acute myocardial infarction is one of the commonest causes of hospitalization and death in the western industrialized countries. It is therefore of great importance to have a fast, inexpensive, sensitive, and specific diagnostic test. The most widely used single laboratory test is electrophoretic determination of the $\mathrm{MB}$ isoenzyme of creatine kinase (EC 2.7.3.2) ${ }^{1}$ ) (creatine kinase-MB) $(1,2)$. This method is very specific and reasonably sensitive, but it is rather tedious and expensive $(3,4)$. The last decade has seen the development of easier analytical methods, which utilize

1) Enzymes

Adenylate kinase EC 2.7.4.3

Creatine kinase EC 2.7.3.2 specific antibodies against the subunit $\mathrm{M}$. The antibodies have either been used in radioimmunoassays or for specific inhibition of the enzymatic activity of the subunit $M$, followed by measurement of the remaining enzymatic activity $(2,5)$. The aim of this study is to compare two different immunoinhibition methods (a dry and a wet chemistry method) with the commonly used but laborious electrophoretic method.

\section{Materials and Methods}

Samples

The study included all consecutive serum samples taken for creatine kinase-MB measurement at Glostrup Hospital during a two-month period: 563 serum samples from 235 patients $(150$ men: mean age 59 years, range $19-90$ years; 85 women: mean age 65 years, range $31-91$ years) 
Measurement of total creatine kinase and creatine kinase-MB activity

\section{Electrophoretic method}

Total creatine kinase activity was measured on an LKB 8600 Rate Analyzer (LKB, Bromma, Sweden), according to the recommendations of the Scandinavian Committee on Enzymes (SCE) $(6,7)$, using a reagent kit (CK NACactivated - Monotest - No. 126357) from Boehringer Mannheim (Mannheim, W. Germany). Serum samples were diluted at total creatine kinase activities above $1400 \mathrm{U} / \mathrm{l}$. Relative creatine kinase-MB activity was measured by fluorometry, using a Vitatron TLD 100 densitometer (Vitatron, Dieren, The Netherlands) after electrophoretic separation on agarose gel film and reaction with substrate (Creatine Phosphokinase Isoenzyme Substrate Set Corning, Halstead, UK) $(2,3)$. By this method the creatine kinase is separated into the three main isoenzymes: creatine kinase-MM, creatine kinase-MB, and creatine kinase- $\mathrm{BB}$. The total creatine kinase activity applied to the agarose gel film did not exceed $1000 \mathrm{U} / 1$ (3). Creatine kinase-MB activity was calculated by multiplying the relative creatine kinase-MB activity by the total creatine kinase activity.

\section{Dry chemistry method}

Measurements were performed on an Ektachem 700 (Eastman Kodak, Rochester, NY). Total creatine kinase activity was measured using Clinical Chemistry Slides (CK - Generation 3) (8) from Eastman Kodak, calibrated against the SCE method $(6,7)$. Serum samples were diluted at total creatine kinase activities above $1600 \mathrm{U} / 1$.

Creatine kinase-MB activity was measured using Clinical Chemistry Slides (CK-MB - Generation 2) (9) from Eastman Kodak, calibrated against the SCE method for measuring creatine kinase- $B$ subunit activity $(5,10)$. The creatine kinase-MB activity is calculated as the measured creatine kinase- $B$ subunit activity multiplied by 2 . Serum samples were diluted at creatine kinase-MB activities above $300 \mathrm{U} / 1$.

\section{Wet chemistry method}

Measurements were performed on a Cobas Mira (Hoffmann La-Roche, Basel, Switzerland). Total creatine kinase was measured according to the SCE method $(6,7)$ using reagents from Hoffmann La-Roche (CK NAC MA-KIT - No. 0713252), following the protocol given by the manufacturer. Serum samples were diluted at total creatine kinase activities above 1000 $\mathrm{U} / \mathrm{l}$. Creatine kinase-MB was measured according to the SCE method for measuring creatine kinase-B subunit activity $(5,10)$ using reagents from Hoffman La-Roche (CK-MB MA-KIT No. 0720666). Serum samples were diluted at creatine kinaseMB activities above $1000 \mathrm{U} / \mathrm{l}$. Creatine kinase-MB activity was corrected for interference from adenylate kinase') (EC 2.7.4.3) by subtracting the activity found before addition of creatine phosphate, as recommended by Gerhardt \& Waldenström (10). Results below zero were given as zero. The creatine kinase-MB activity was calculated as the measured creatine kinase-B subunit activity multiplied by 2 .

All dilutions were made using saline.

\section{Detection limit}

The detection limit was estimated as the mean of ten measurements of the activity of a heat inactivated sample $+3 \mathrm{SD}$.

\section{Precision}

Intra- and inter-assay precisions were estimated on a serum sample analysed in duplicate in nine separate runs. The serum sample was kept at $-80^{\circ} \mathrm{C}$ until assayed.
Diagnostic assessment

All serum samples from each patient were assessed as a group and, if one sample from a patient complied with the criteria, the patient was categorised as having acute myocardial infarction. The criterion used for the electrophoretic method was: creatine kinase-MB $\geqslant 30 \mathrm{U} / 1$ (3). For the dry chemistry method and wet chemistry method, two approaches were made:

A: Application of the criteria recommended for the individual method:

Dry chemistry method:

total creatine kinase $\geqslant 65 \mathrm{U} / 1$,

creatine kinase-MB $\geqslant 16 \mathrm{U} / \mathrm{l}$, and

$5 \% \leqslant$ creatine kinase-MB fraction $\leqslant 24 \%$

Wet chemistry method:

total creatine kinase $\geqslant 420 \mathrm{U} / 1$, creatine kinase-MB $\geqslant 25 \mathrm{U} / \mathrm{l}$, and

creatine kinase-MB fraction $\geqslant 6 \%$ (according to manufacturer's recommendation)

B: Application of the criteria found by optimizing efficiency (12) for the three discriminatory limits simultaneously:

The optimization of efficiency was performed for the following range of discriminators: total creatine kinase $\geqslant 50-400 \mathrm{U} / \mathrm{l}$ in steps of $50 \mathrm{U} / \mathrm{l}$, creatine kinase-MB $\geqslant 10-40 \mathrm{U} / \mathrm{l}$ in steps of $5 \mathrm{U} / 1$, and creatine kinase-MB fraction $\geqslant 0-7 \%$ in steps of $1 \%$. The upper limit of creatine kinase-MB fraction was maintained at $25 \%$

\section{Estimation of infarct size}

Finally the electrophoretic method and dry chemistry method were used to estimate infarct size (13) in eight patients with acute myocardial infarction. Creatine kinase-MB was measured by both methods in $9-11$ serum samples taken from each patient over two days (about every four hours).

\section{Statistics}

The statistics were computed with a STATGRAPHICS program (STSC, Rockville, MD) for larger data series. Precision errors were calculated by standard statistical procedures.

Deming regression was used to compare the methods $(14,15)$. Only undiluted samples were used in the comparison, so as to avoid bias due to dilution and skewness in weighting of extreme values. This, however, resulted in different numbers of observations, because the dilution limits varied between the methods.

\section{Results}

\section{Methodological assessment}

\section{Total creatine kinase}

As shown in table 1, the precision of all three methods is comparable. The three methods were highly correlated:

Electrophoretic method $=0.95 \times$ dry chemistry method $+12 \mathrm{U} / 1$

$\left.(\mathrm{n}=505, \mathrm{r}=0.994, \mathrm{SEE}=27 \mathrm{U} / \mathrm{l}){ }^{2}{ }^{2}\right)$

\footnotetext{
$\left.{ }^{2}\right) \mathrm{n}$ : number of observations

r: correlation coefficient

SD: standard deviation

SEE: standard error of estimate

n. s.: non significant
} 
Tab. 1. Intra- and inter-assay precision for total creatine kinase and creatine kinase-MB determinations estimated for serum sample measured in duplicate in separate runs.

\begin{tabular}{|c|c|c|c|c|c|c|c|}
\hline & \multirow[t]{2}{*}{$\mathrm{n}$} & \multicolumn{3}{|c|}{ Creatine kinase $(500 \mathrm{U} / \mathrm{l})$} & \multicolumn{3}{|c|}{ Creatine kinase-MB (40 U/l) } \\
\hline & & $\begin{array}{l}\text { Electro- } \\
\text { phoretic } \\
\text { method }\end{array}$ & $\begin{array}{l}\text { Dry } \\
\text { chemistry } \\
\text { method }\end{array}$ & $\begin{array}{l}\text { Wet } \\
\text { chemistry } \\
\text { method }\end{array}$ & $\begin{array}{l}\text { Electro- } \\
\text { phoretic } \\
\text { method }\end{array}$ & $\begin{array}{l}\text { Dry } \\
\text { chemistry } \\
\text { method }\end{array}$ & $\begin{array}{l}\text { Wet } \\
\text { chemistry } \\
\text { method }\end{array}$ \\
\hline Intra-assay CV\% & 9 & 1.8 & 0.8 & 1.2 & 4.3 & 4.3 & 3.5 \\
\hline Inter-assay CV\% & 9 & 2.3 & 2.2 & 2.0 & 7.8 & 4.3 & 7.7 \\
\hline
\end{tabular}

$\mathrm{n}$ : Number of duplicate runs.

Electrophoretic method $=0.92 \times$ wet chemistry method $+5 \mathrm{U} / 1$

$(\mathrm{n}=487, \mathrm{r}=0.996, \mathrm{SEE}=16 \mathrm{U} / \mathrm{l})$.

The same linear relation was found when the diluted samples were included (data not shown).

Using the heat-inactivated sample, the detection limit was found to be $3.9 \mathrm{U} / 1$ for the wet chemistry method, and below the lower limit of the manufacturer's fixed measuring range of $1 \mathrm{U} / 1$ for the dry chemistry method.

\section{Creatine kinase- $M B$}

As shown in table 1, the inter-assay precision of the dry chemistry method was slightly better, whereas the intra-assay precision of all three methods was comparable.

Figure 1 ( $a$ and $b$ ) compares the methods for measuring creatine kinase-MB. Plots of the electrophoretic method against the dry chemistry method and the wet chemistry method show slopes significantly higher than unity and intercepts significantly lower than zero.

The correlation coefficient was found to be larger $(p \leqslant 0.001)$ and the $\mathrm{SEE}^{2}$ ) smaller for the dry chemistry method than for the wet chemistry method. The same linear relation was found when the diluted samples were included (not shown). As noted in figure 1 two samples showed large deviations from the other methods by the wet chemistry method. These results were grave methodological errors as they could not be reproduced by reanalysis.

Using the heat-inactivated sample, the detection limit was found to be $6.6 \mathrm{U} / 1$ for the wet chemistry method and below the lower limit of the manufacturer's fixed measuring range of $1 \mathrm{U} / 1$ for the dry chemistry method.
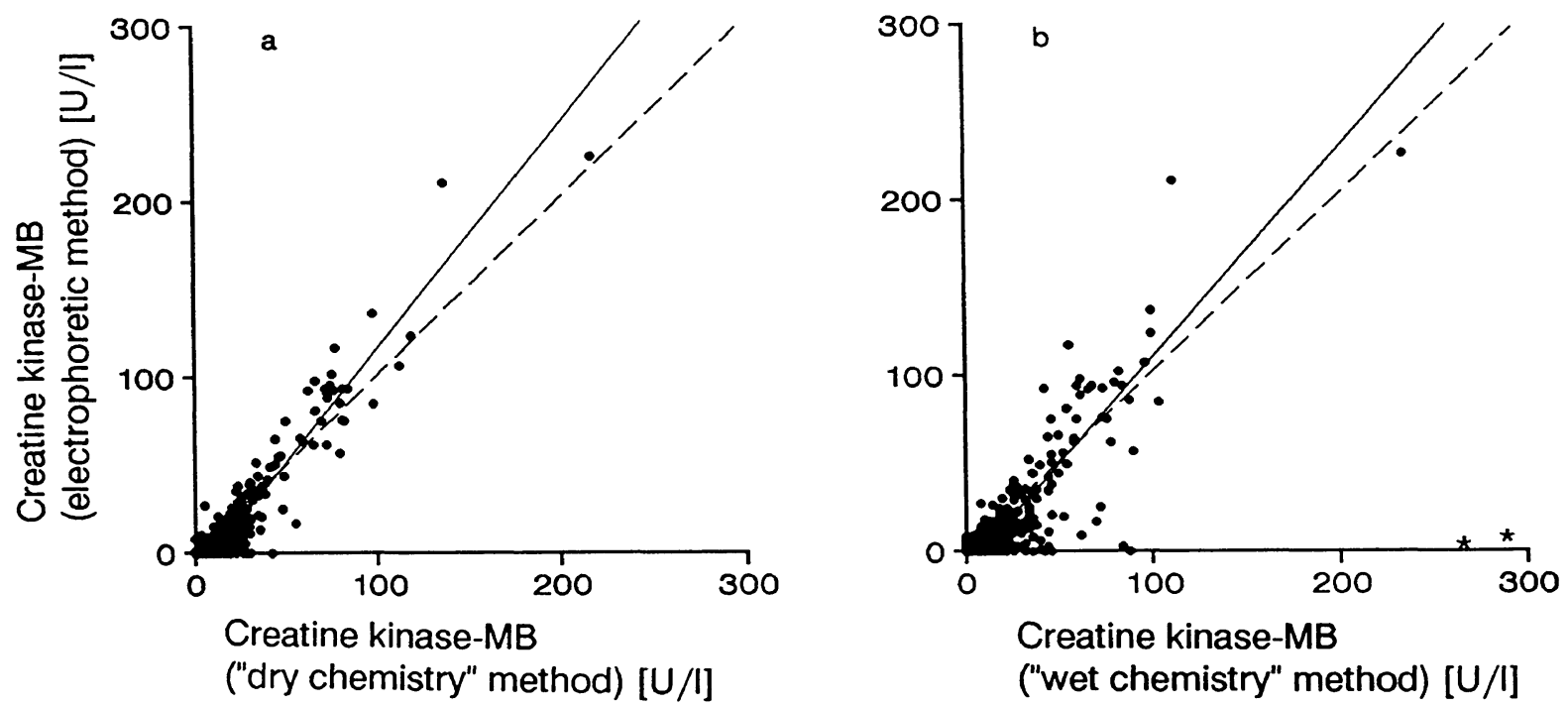

Fig. 1. Comparison of methods for determination of creatine kinase-MB by Deming regression. Assessment performed on undiluted serum samples.

$(-)$ Regression line, $(---)$ Identity line.

a: Electrophoretic method $=1.22 \times$ dry chemistry method $-6.5 \mathrm{U} / 1(\mathrm{n}=513, \mathrm{r}=0.943, \mathrm{SEE}=8.4 \mathrm{U} / \mathrm{l})$

b: Electrophoretic method $=1.19 \times$ wet chemistry method $-7.2 \mathrm{U} / \mathrm{l}(\mathrm{n}=511, \mathrm{r}=0.854, \mathrm{SEE}=13.5 \mathrm{U} / \mathrm{l})$.

In the calculation of regression $b$ parameters, two results that deviated more than five SEE from the regression line were excluded from the calculation (indicated by * in the figure). 


\section{Creatine kinase- $B B$}

The influence of creatine kinase-BB was investigated in a random sample consisting of 199 serum samples from 79 of the 235 patients. Fifteen $(7 \%)$ of these samples originating from $13(10 \%)$ patients had creatine kinase- $\mathrm{BB} \geqslant 5 \mathrm{U} / \mathrm{l}: 9.1 \pm 2.6 \mathrm{U} / \mathrm{l}$ (mean $\pm \mathrm{SD}$ ). However, this only resulted in an overestimation of creatine kinase-MB of $5.3 \pm 7.2 \mathrm{U} / 1$ by the dry chemistry method and of $7.9 \pm 7.0 \mathrm{U} / 1$ by the wet chemistry method.

\section{Adenylate kinase}

In the presence of haemolysis equivalent to the release of $50 \mathrm{mmol} / \mathrm{l}$ haemoglobin, the erythrocyte-derived adenylate kinase caused no significant interference in the creatine kinase measurement by any of the three procedures (data not shown).

\section{Diagnostic assessment}

Of the 235 patients, 36 were categorized by the electrophoretic method as having acute myocardial infarction, which gives a prevalence of $15 \%$. Comparison of the dry chemistry method and wet chemistry method with the electrophoretic method using approach $A$ (tab. 2) showed that the dry chemistry method and wet chemistry method were almost equally efficient, but that the wet chemistry method had a rather low sensitivity. Altogether 24 patients were classified differently than by the electrophoretic method. Three false negatives were found by the dry chemistry method and the wet chemistry method, because the creatine kinase-MB fraction was below the given limits.

The false negatives found by the wet chemistry method are chiefly explained by the high total creatine kinase limit and creatine kinase-MB fraction limit.
The dry chemistry method showed 11 false positives. In five cases the electrophoretic method and dry chemistry method yielded similar values, but indicated different diagnoses, because of the different discriminators of the two methods. Five more had increased values, owing to interference from creatine kinase- $\mathrm{BB}$ or macro creatine kinase, and in one the cause was analytical error (found to be normal on re-analysis).

The optimal discriminators found by approach $B$ for the dry chemistry method were: total creatine kinase $\geqslant 100 \mathrm{U} / 1$, creatine kinase-MB $\geqslant 25 \mathrm{U} / 1$ and $4 \% \leqslant$ creatine kinase-MB fraction $\leqslant 25 \%$; and for the wet chemistry method: total creatine kinase $\geqslant 350 \mathrm{U} / \mathrm{l}$, creatine kinase-MB $\geqslant 35 \mathrm{U} / 1$ and $4 \% \leqslant$ creatine kinase-MB fraction $\leqslant 25 \%$. Approach B clearly showed better performance characteristics (tab. 2) than did approach $\mathrm{A}$, as only three patients were classified differently by the electrophoretic and the dry chemistry methods, only four by the electrophoretic and the wet chemistry methods.

\section{Estimation of infarct size}

Infarct size was estimated from the area under the curve of serum creatine kinase-MB as a function of time. Comparison of estimated infarct size by the dry chemistry and electrophoretic methods showed the relationship: electrophoretic method $=1.25 \times$ dry chemistry method +1.4 (n.s.) for 8 patients giving $r=0.992$ and relative SEE of $9 \%$.

\section{Discussion}

When the present data are compared with those reported in earlier assessments of the immunoinhibition methods and the electrophoretic method, details of reagent composition, sample volume, and temperature are of paramount importance $(1,2,6,7)$. These

Tab. 2. Assessment of immunoinhibition methods in the diagnosis of acute myocardial infarction as established by the electrophoretic method:

\begin{tabular}{|c|c|c|c|c|c|c|c|c|c|}
\hline \multirow[b]{3}{*}{$\begin{array}{l}\text { Electrophoretic } \\
\text { method }\end{array}$} & \multirow[b]{3}{*}{$\begin{array}{l}\text { pos. } \\
\text { neg. }\end{array}$} & \multicolumn{4}{|c|}{ Approach A } & \multicolumn{4}{|c|}{ Approach B } \\
\hline & & \multicolumn{2}{|c|}{ Dry chemistry method } & \multicolumn{2}{|c|}{ Wet chemistry method } & \multicolumn{2}{|c|}{ Dry chemistry method } & \multicolumn{2}{|c|}{ Wet chemistry method } \\
\hline & & $\begin{array}{r}\text { neg. } \\
3 \\
188\end{array}$ & $\begin{array}{l}\text { pos. } \\
33 \\
11\end{array}$ & $\begin{array}{r}\text { neg. } \\
12 \\
198\end{array}$ & $\begin{array}{c}\text { pos. } \\
24 \\
1\end{array}$ & $\begin{array}{r}\text { neg. } \\
2 \\
198\end{array}$ & $\begin{array}{c}\text { pos. } \\
34 \\
1\end{array}$ & $\begin{array}{r}\text { neg. } \\
3 \\
198\end{array}$ & $\begin{array}{c}\text { pos. } \\
33 \\
1\end{array}$ \\
\hline Sensitivity & & \multicolumn{2}{|c|}{0.92} & \multicolumn{2}{|c|}{0.67} & \multicolumn{2}{|c|}{0.94} & \multicolumn{2}{|c|}{0.92} \\
\hline Specificity & & \multicolumn{2}{|c|}{0.94} & \multicolumn{2}{|c|}{0.99} & \multicolumn{2}{|c|}{0.99} & \multicolumn{2}{|c|}{0.99} \\
\hline Predictive value pos. & & \multicolumn{2}{|c|}{0.75} & \multicolumn{2}{|c|}{0.96} & \multicolumn{2}{|c|}{0.97} & \multicolumn{2}{|c|}{0.97} \\
\hline Predictive value neg. & & \multicolumn{2}{|c|}{0.98} & \multicolumn{2}{|c|}{0.94} & \multicolumn{2}{|c|}{0.99} & \multicolumn{2}{|c|}{0.98} \\
\hline Efficiency & & \multicolumn{2}{|c|}{0.94} & \multicolumn{2}{|c|}{0.95} & \multicolumn{2}{|c|}{0.99} & \multicolumn{2}{|c|}{0.98} \\
\hline
\end{tabular}

Assessment on 235 patients, 36 of whom had acute myocardial infarction according to the electrophoretic method, which was equivalent to a prevalence of $15 \%$. 
parameters influence the purely methodological comparison, because they affect the slopes, and they also influence the diagnostic assessment, because the discriminators might change.

The technical performance of the three total creatine kinase methods is good and the precisions are acceptable. The larger SEE for the dry chemistry method can probably be ascribed to a higher sensitivity to matrix effects (16). The precision for creatine kinase-MB is consistent with earlier investigations (3, $5,10,11,17)$. The detection limits for the wet chemistry methods were rather high, especially for creatine kinase-MB. This was partly due to the correction for a negative adenylate kinase activity, but the mean creatine kinase-MB activity alone was $3.4 \mathrm{U} / 1$, giving a detection limit of $4.9 \mathrm{U} / 1$. When measured by the dry chemistry method the heat-inactivated sample gave activities below the lower limit of the manufacturer's fixed measuring range of $1 \mathrm{U} / \mathrm{l}$.

As seen in figure 1 ( $a$ and $b$ ), both the dry chemistry method and the wet chemistry method gave lower values for high activity samples and higher values for low activity samples, as compared with the electrophoretic method $(17,18)$.

This phenomenon is probably caused by two different factors. It is well-known that in the electrophoretic method the fluorescence at higher intensity is quenched $(2,11)$. As the creatine kinase-MM fraction is always greater than the creatine kinase-MB fraction this will cause overestimation of creatine kinase-MB activity (11). On the other hand, the immunoinhibition assays may overestimate creatine kinase-MB activity in some low activity samples, owing to interference from even small amounts of creatine kinase-BB and macro creatine kinase activity in this activity range $(5,19)$.

The number of samples showing creatine kinase-BB activity was larger than reported earlier (19), possibly because of differences in sample handling, as creatine kinase-BB activity is quickly inactivated at room temperature (19). This might also explain the discrepancy between the estimate of creatine kinase-BB activity found by electrophoresis and that found by the immunoinhibition methods, as these were performed after the electrophoretic separation was started. The possibility, however, that fluorescent substances interfered $(1,2)$ cannot be ruled out, as a control incubation without creatine phosphate was not performed.

The accuracy of the methods was estimated by comparison with the electrophoretic method; various lyophilized materials with assigned values were inappro- priate for analysis by the dry chemistry method, because their viscosity differed from that of serum (16).

The diagnosis of acute myocardial infarction is based on three criteria: pathological electrocardiographic changes, acute chest pain, and increased creatine kinase-MB activity. We chose to assess the new immunoinhibition methods against the electrophoretic method alone, because it is well established as the optimal single diagnostic test in patients with suspected acute myocardial infarction $(20,21)$.

We found that when we applied the manufacturer's diagnostic criteria, the dry chemistry method gave too many false positives and the wet chemistry method too many false negatives. The acceptable balance between high sensitivity and high specificity will always depend on the population involved (12). Because of the severity of the disease, high sensitivity is preferable to high specificity.

We found that the creatine kinase-MB limit for the dry chemistry method could be raised to $25 \mathrm{U} / 1$ without misclassifying more than one patient with acute myocardial infarction. The reason for the low creatine kinase-MB limit might be the use of single samples in earlier assessments. We used the information gathered from a series of samples from each patient suspected of acute myocardial infarction.

The wet chemistry method gave too many false negatives, mainly because of the very high total creatine kinase limit recommended by the manufacturer, which is higher than other recommendations $(5,22)$. The creatine kinase-MB fraction limit should also be lowered. The value of $6 \%(22)$ is probably based on older methods, either with activators other than $\mathrm{N}$-acetyl cysteine (NAC) or without correction for interference from adenylate kinase $(5,10)$.

The optimal discriminators found for the two immunoinhibitory methods were rather different. The main reason for the high values for the wet chemistry method were a few samples from patients categorized as having no myocardial infarction, with creatine kinase-MB activities in the range of $25-50 \mathrm{U} / 1$. A change of the creatine kinase-MB discriminator to 25 $\mathrm{U} / 1$ thus only reduced the specificity to 0.98 . The creatine kinase discriminator could be changed simultaneously to $100-200 \mathrm{U} / 1$ without affecting the specificity further. For the dry chemistry method a change of the creatine kinase discriminator to $300 \mathrm{U} / 1$ showed unchanged efficiency, but a sensitivity of 0.92 and a specificity of 1.0 .

Application of the lower creatine kinase-MB fraction limit, with a view to eliminating false positive results arising from either incomplete inhibition of creatine 
kinase-MM activity (5) or creatine kinase-MB activity from skeletal muscle damage $(1,4)$, has been discussed by others $(10,23)$. The disadvantage is that an acute myocardial infarction could be masked by a simultaneous major release of creatine kinase-MM from non-myocardial sources.

We have earlier shown that the electrophoretic method may be used to estimate infarct size (13). Our data clearly demonstrate that similar results would be obtained with the dry chemistry method. From the present study it can be concluded that the immunoinhibition methods possess performance characteristics comparable to those of the well-known electropho- retic method. The chief advantages are ease of use and speed. The chief disadvantage is the lack of specificity against creatine kinase-BB and macro creatine kinase which, however, seems to be a minor problem. The use of optimized discriminators is, however, recommended for a given patient population, because the efficiency optimization is only valid for populations with a similar disease prevalence.

\section{Acknowledgement}

We thank Eastman Kodak Co. for kindly providing the slides for the present investigation.

\section{References}

1. Bais, R. \& Edwards, J. B. (1982) Creatine kinase [Review]. Crit. Rev. Clin. Lab. Sci. 16, $291-335$.

2. Foreback, C. C. \& Chu, J.-W. (1981) Creatine kinase isoenzymes: Electrophoretic and quantitative measurements [Review]. Crit. Rev. Clin. Lab. Sci. 15, 187-230.

3. Grande, P., Christiansen, C. \& Næstoft, J. (1979) Creatine kinase isoenzyme MB assay by electrophoresis. Scand. J. Clin. Lab. Invest. 39, 607-612.

4. Lott, J. A. (1984) Serum enzyme determinations in the diagnosis of acute myocardial infarction: An update [Review]. Hum. Pathol. 15, 706-716.

5. Scandinavian Committee on Enzymes (1981) Creatine kinase (EC 2.7.3.2) and creatine kinase B-subunit activity in serum in suspect myocardial infarction. The Nordic Clinical Chemistry Project (NORDKEM), Helsinki, Finland.

6. Scandinavian Committee on Enzymes (1976) Recommended method for the determination of creatine kinase in blood. Scand. J. Clin. Lab. Invest. 36, 711-723.

7. Scandinavian Committee on Enzymes (1979) Recommended method for the determination of creatine kinase in blood modified by the inclusion of EDTA. Scand. J. Clin. Lab. Invest. $39,1-5$.

8. Findlay, J. B., Searle, R., Thunberg, A. \& Schubert, R. (1983) Development of a Kodak Ektachem clinical chemistry slide for creatine kinase [Abstract]. Clin. Chem. 29, 1212.

9. Findlay, J. B., Wu, A. L., Knott, V., Mauck, L., Frickey, H. \& Norton, G. E. (1985) Development of a Kodak Ektachem clinical chemistry slide for creatine kinase-B activity [Abstract]. Clin. Chem. 31, 1000.

10. Gerhardt, W. \& Waldenström, J. (1979) Creatine kinase Bsubunit activity in serum after immunoinhibition of $M$ subunit activity. Clin. Chem. 25, 1274-1280.

11. Kwong, T. (1986) Evaluation of the Kodak Ektachem clinical chemistry slide for creatine kinase-MB. Wisconsin Kodak Users' Club seminar, Pewaukee, Wisconsin, October 30, 1986.

12. Gerhardt, W. \& Keller, H. (1986) Evaluation of test data from clinical studies. Scand. J. Clin. Lab. Invest. 46 suppl. 181.

13. Grande, P., Næstoft, J. \& Christiansen, C. (1980) An easy and reliable estimation of acute myocardial infarct size from serum creatine kinase-MB measurements. Eur. J. Cardiol. $11,71-77$.

14. Mandel, J. (1964) The statistical analysis of experimental data, New York: Interscience pp. 288-292.

15. York, D. (1966) Least-squares fitting of a straight line. Can. J. Physics. 44, 1079-1086.

16. Greyson, J. (1981) Problems and possibilities of chemistry on dry reagent carriers. J. Aut. Chem. 3, 66-71.

17. Grande, P. \& Christiansen, C. (1983) Clinical evaluation of different principles of creatine kinase-MB determination. Scand. J. Clin. Lab. Invest. 43, 197-201.

18. Doria Jr, M., Bissell, M., Doria, L., Sanghavi. P., Ward, E., Powell, D., Isidra, E., Toress, F., Baron, S. \& Harrison, H. (1988) Evaluation of the Kodak Ektachem slide method for creatine kinase-MB [Abstract]. Clin. Chem. 34, 1284.

19. Lang, H. \& Würzburg, U. (1982) Creatine kinase, an enzyme of many forms [Review]. Clin. Chem. 28, 1439-1447.

20. Grande, P., Christiansen, C., Pedersen, A. \& Christensen, M. S. (1980) Optimal diagnosis in acute myocardial infarction. A cost-effectiveness study. Circulation 61, 723-728.

21. Ljungdahl, L., Hofvendahl, S., Gerhardt, W. \& Börjesson, J. (1977) Creatine kinase B-subunit activity in human serum. II. Evaluation of S-creatine kinase B-subunit activity in the diagnosis of acute myocardial infarction. Clin. Chim. Acta $78,43-53$.

22. Neumeier, D., Prellwitz, W. \& Knedel, M. (1981) Creatine kinase isoenzymes after myocardial infarction. In: Creatine kinase isoenzymes (Lang, H., ed.) pp. 132-154, SpringerVerlag Berlin Heidelberg New York.

23. Gerhardt, W., Waldenström, J., Hörder, M., Hofvendahl, S., Billstrøm, R., Ljungdahl, R., Berning, H. \& Bagger, P. (1982) Creatine kinase and creatine kinase B-subunit activity in serum in cases of suspected myocardial infarction. Clin. Chem. 28, 277-283.

Aksel Hadberg

Department of Clinical Chemistry

Glostrup Hospital

University of Copenhagen

DK-2600 Glostrup 